\title{
Model Based Processing of Swabbing Movements on Touch Screens to Improve Accuracy and Efficacy for Information Input of Individuals Suffering from Kinetic Tremor
}

\author{
A. Mertens ${ }^{1}$, C. Wacharamanotham ${ }^{2}$, J. Hurtmanns ${ }^{1,2}$, M. Kronenbuerger ${ }^{3}$, \\ P.H. Kraus ${ }^{4}$, A. Hoffmann ${ }^{4}$, C. Schlick ${ }^{1}$ and J. Borchers ${ }^{2}$ \\ ${ }^{1}$ Institute of Industrial Engineering and Ergonomics, RWTH Aachen University, Germany, \\ \{a.mertens, j.hurtmanns, c.schlick\}@iaw.rwth-aachen.de \\ ${ }^{2}$ Media Computing Group, RWTH Aachen University, Germany, \\ \{chat, borchers\}@cs.rwth-aachen.de \\ ${ }^{3}$ Department of Neurology, RWTH Aachen University, University Hospital Aachen, Germany, \\ mkronenbuerger@ukaachen.de \\ ${ }^{4}$ Department of Neurology, Ruhr-University Bochum, St. Josef-Hospital, Germany, \\ \{peter.h.kraus, arndt.hoffmann\}@ruhr-uni-bochum.de
}

\begin{abstract}
As a result of demographic change the average age of many western populations increases, accompanied with age-related disease patterns. Especially tremor symptoms rise accordingly, aggravating a barrier free interaction with information systems. In order to maintain a self determined lifestyle at home, new technologies and methods need to be introduced, especially for application in health care and telemedical scenarios. Hence, a new direct input technique based on wiping movements on touch screens has been developed. The combination of a new input concept and applying regular commercially available technologies helps to avoid high costs for acquisition and therefore makes it marketable. While making an input on the touch screen the precise characteristics of every wiping movement can be tracked and is used for computation of the desired entry. The efficacy of this approach was evaluated within a clinical study with $n=15$ subjects. The results show that the error ratio for inputs by tremor patients can be significantly reduced in comparison to a virtual keyboard, depending on tremor strength and form. The learning curve for first time users is very steep and tends to result in inputs that are only slightly steady than purposeful movements to standard buttons and keys.
\end{abstract}

\section{Introduction}

\subsection{Motivation}

Strong tremor symptoms increase the error rate of human-computer interaction and may even lead to a total refusal or inability to utilize an IT-based system for 
communicating individual needs. The currently available systems do not service people suffering from kinetic tremor. Those tremor patients often suffer from a level of high inaccuracy and show a worsening of precision when moving towards a virtual or real button, due to the nature of intention tremor. This inability abates efficiency, effectiveness and satisfaction of the user and even causes social isolation due to the hindered maintaining and establishing of contacts [Martínez-Martín 1998]. For this determined target group a new method of interaction has been developed and tested. Creating a sufficient interaction for people with a tremor agitation using the existing interface hardware is the key to several identified barriers occurring with the current demographic change and augments the potentials for developers. Optimizing current technology will help to maintain a personal health care system and retain the trust in it. Creating an interaction which allows user to effectively use their tremor encumbrance as part of their interaction process is highly worthwhile.

The correlation of efficiency and satisfaction for the elderly during computer based interaction has already been proven. As user acceptance, among elderly, towards new technologies is considered mediocre at best, it will help to quickly establish a user-interface relationship if the user recognizes his natural motion pattern as a required input.

\subsection{Tremor in Older Adults}

The word tremor is derived from Latin tremere, meaning „to tremble“. Tremor can be defined as involuntary oscillations of any part of the body around any plane, such oscillations being either regular or irregular in rate and amplitude and resulting from alternate or synchronous actions of groups of muscles and their antagonists. Arms are the part of the body that is most commonly affected. Tremors can happen irrespective of age but show a tendency to be prevalent in older people. About $1-4 \%$ of the total population is concerned while in the population group older than 50 years up to 14.5 are afflicted [Wenning et al. 2005]. Tremors are usually classified according to their phenomenology, most commonly "present at rest" or "present with action" (i.e. posture or movement). Occurrence and intensity can differ highly for each person affected based on physical and mental state of the day and medication. The frequency varies depending on etiologies with a range between 3 and $30 \mathrm{~Hz}$. The most common action tremor is the so called "Essential Tremor".

\subsection{Classification of Tremor Strength}

The quantification of motor performance of people afflicted with tremor is usually assisted with help of clinical rating scales and instrumental approaches that allow estimating the efficacy of applied therapy. While manual rating scales often are 
not sufficient objective the usage and appraisal of instrumented methods is mostly restricted to hospitals and medical specialists. Therefore a novel approach based on automated rating tremor strength with help of spiral drawings is applied within this study (see chapter 4.3 for details). This instrument allows a time and location independent application by non-physicians and guarantees high reliability because of the standardized interpretation by a computer-algorithm. Focus is just on quantification of tremor amplitude, regardless of clinical diagnosis and independent of tremor genesis.

\subsection{Requirements for Information Input by People Suffering from Tremor}

To allow a satisfying and effective interaction of people suffering from tremor with IT-systems, especially the information input has to be focused as prevalent user interfaces do not respect the specific needs and limitations. To get an overview of the different tremor pathologies and the manifold effects they have on activities of daily living, field observations and expert interviews were accomplished. Result was the deeper insight that the impact very much depends on living conditions, adequate assistance and symptomatology which can be seen in the exemplarily handwritings presented in Figure 1.

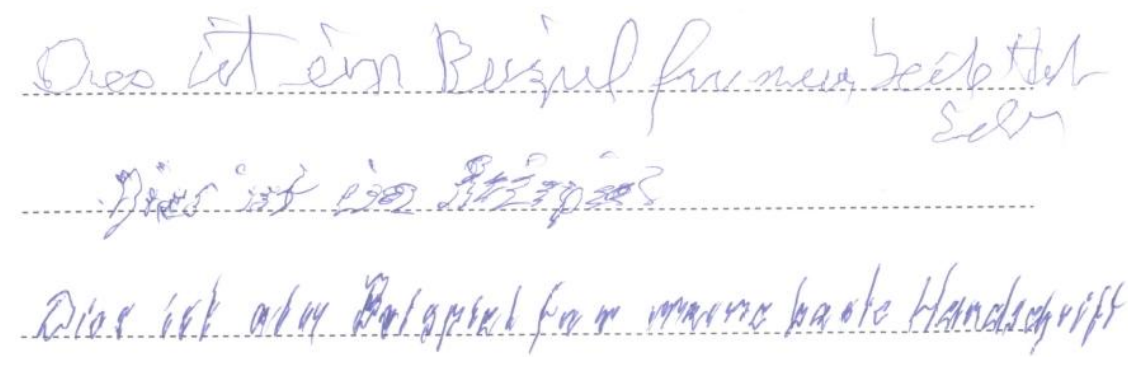

Fig. 1 Exemplary handwritings of individuals suffering from different tremor types

Workshops with the people affected by tremor, medical specialists and medical technicians resulted in several requirements that have to be satisfied by potential manual input concepts to be adequate utilities:

- Input must be identified as one gesture even when the contact while interacting with the device is lost for a short time because of jerks in zaxis

- Fault insertions because of unintentional contacts and laying down the palm have to be filtered out

- The input concept must me adaptive to the varying tremor strength depending on the form of the day as well as medication and not be depending on a calibration 
- Different phenomenologies of tremor must be considered including tremor amplification while resting and with action

- Recognition of selections for non linear input patterns (e.g. staggered or sinuous actions)

\subsection{Related Research}

There has been a rapid development in the domain of human-computer interfaces over the past two decades. Among them are technical innovations and enhancements as motion and eye tracking devices, voice recognition and brain-computer interfaces allowing a new dimension of information input. A possible application of the devices includes everyday support for physically impaired persons. However, there are several limitations to the use of these technologies with regard to mobility, costs, privacy protection, noise immunity, training time and required calibration. These criteria constrain a broad distribution and allow feasibility only for very specific scenarios. A wide use in particular for the growing number of elderly people with limited motor skills is not realistic in the medium term.

Therefore research on the budding application of touch screens as input medium for tremor patients was accomplished. This technology has evolved from a niche product to the quasi standard input of smartphones and can already be found in e.g. ready-made computer systems, ATMs, route guidance systems and ticket machines. It turned out to be a relative untouched research field from an ergonomic point of view and with regard to the identified requirements. Research has mostly been done for medical questions concerning the rating and categorizing of tremor symptoms for different treatments and therapies.

To have a valid foundation for the development of an advanced input technique and to not reinvent the wheel, an in detail literature review was accomplished. The following section provides an overview of some "classic" approaches and concepts towards input techniques with touch screens that were appraised.

\section{Buxton's three state model}

Buxton (1990) distinguishes three states of input: (0) out of range, (1) tracking and (2) dragging. Illustrated with mouse usage, state zero is when the mouse is not in contact with any surface (i.e. moving the mouse has no input effect); state one refers to the mouse being moved on a surface (i.e. the cursor moves according to mouse movements); finally, when selecting a target (i.e. pressing a button), the target can be moved around the screen. However, applying this model to touch screens results in complete omission of stage 1; instead, the system jumps directly from state zero to state two. The reduction to only two states brings about advantages in terms of input time, although not necessarily reducing error rates.

In addition to Buxton's three stages, Potter et al. (1988) divide target acquisitions on touch screens into three different strategies: Land on, First contact and Takeoff. Input methods utilizing the Land on strategy make their selection based on the 
first initial contact with the screen; all further contacts are ignored. First contact strategies are similar to Land on strategies, "but take advantage of the continuous stream of touch data. This means that the first target which is hit will also be selected.

Take-off input strategies utilize a curser, which is moved by dragging the finger on the screen (as in First contact) but the selection is only made when the finger is removed from the screen.

Offset Cursor (First contact)

In order to avoid target occlusion by the finger, Potter et al. designed an alternative input method called Offset Cursor. When the finger is placed on the display, the selection is not made at the point of contact; instead a curser appears above the finger and allows for precise selection of targets by dragging towards the wished location.

As much as it improves the problem of target occlusion, the main disadvantage of Offset Cursor is that the selection is always performed above the finger, making targets at the bottom area of the screen impossible to select.

Direct Touch (Land on)

Direct Touch is the most basic and straightforward input for touch screens. The principle of this technique is very intuitive: the user selects a target on the screen by directly tapping on the location of the target. In terms of selection time, Direct Touch has proved to be superior as opposed to traditional mouse. On the other hand, when the display, and accordingly the target, is small, Direct Touch results in higher selection errors due to the occlusion of the target by the finger.

Another disadvantage of Direct Touch is the limited accessibility of targets that are located close to each other: the finger occludes the targets and the width of the finger does not allow for accurate input. Further, targets that are located at the border of the screen are difficult to select as well.

TapTap (Land on)

TapTap by Roudaut et al. (2008)] is a technique that is very effective in selecting targets on small tactile displays, e.g. handhelds. The basic idea is that a first tap in the area of the target brings about an enlarged popup of this very area in the middle of the screen, where subsequently the selection is made. TapTap is an especially advanced and effective technique; unlike the Direct Touch input method, TapTap does not that much suffer from thumb occlusion because the user lifts his finger off the screen before making the final selection. In addition to that all areas of the display are equally well accessible.

However, due to the fact that a selection is based on two contacts with the surface, this technique is not as fast as others regarding input time. (Not much information is provided on how to cancel an unwanted selection or whether selecting a target at the edge of the screen interferes with scrolling.) 


\section{MagStick (Take-off)}

Another technique proposed by Roudaut et al. is MagStick. MagStick works in the following way: the user touches the screen in the area of the target he wants to select and then drags his finger in the opposite direction of the target (mirror principle).

The targets work as "magnets" so that the curser automatically jumps to the predefined targets as soon as the finger is moved in the opposite direction. A thin line indicates which target is selected at any given moment and the finger does not occlude the target at any point. Also the selection of targets that are located at the borders of the screen is possible without any restrictions.

\section{Shift (Land on)}

To address the problem of occlusion in bare finger operated touch screen inputs, the Shift method has been proposed as one solution. When targets are small and therefore occluded by the fingers, the occluded area is duplicated and projected to a free region of the screen. When targets are big enough that occlusion is not a problem, this technique is not applied and the display remains unaltered.

However Shift cannot be used when targets are located at the edge of the screen. Studies show that Shift results in faster input times and lower error rates than similar techniques, e.g. the Offset Cursor.

\section{Escape (Take-off)}

Yatani et al. [Yatani et al. 2008] present a target selection technique for mobile displays. The main advantage of Escape is that the user only needs to select a point near the target and then, by moving the finger into the desired direction, can select the target.

In essence, Escape works in the same manner as MagStick. This has proven itself to be extremely useful in selection of small targets. Yatano et al. compare the Escape technique to its alternative Shift and finds that for small targets (size between 6 and 12 pixels) selection is on average $30 \%$ faster while there is no difference in error rates.

\section{Synopsis}

The review of different input techniques regarding touch screens revealed that, although there are several approaches that try to compensate for the disadvantage of others (e.g. thumb occlusion, difficult or impossible to reach areas), none of the discussed methods is promising for elderly people suffering from kinetic tremor or physical impairments of the neuro-musculoskeletal system. The lack of this adaption justifies the need for the development of a enhanced technique that addresses the specific requirements of this target group. 


\subsection{Solution Concept}

\section{Design Pattern: SWABBING}

Problem: Theoretically the problem occurring with a kinetic tremor, namely the inaccurate input, may be handled by simply increasing the size made available on the input area (paper, touch screen, etc...). This would compensate the expected deviation caused by the tremor. However, this method has clear limitations when it comes to stronger tremor deviations and limitation of input space. This leads to either the limitation of options concurrently displayed or the reduction of button size on the screen, in order to maintain the amount of choices offered. This will almost certainly increase the error ratio when handling input made with distinct tremor symptoms and resulting deviation.

Solution: In order to enable correct and independent input for the previously described target group (with smartphones, PC, telemedical systems, ticketing machines - each with a touch screen) the user user interface will be virtually enlarged by using the same area for information input/output and not restricting the input movement to the screen surface. The principle behind the enlargement is Fitt's Law. The width of the target, measured along the axis of motion on the screen, is not restricted through the screen dimensions, characterized in that the user can perform a continuous input movement beyond the borders.

This basically means that all variations or deviations are included and give the user a "free hand" to perform his input. The electronic tracking of the input appears only on the touch screen, but vital data for reproducing the movement, as direction, orientation, velocity and starting point, are collected. This very close approximation of the user movement helps to allocate the desired input from the user much more reliably than the ordinary point input method. Here only the last phase of the input movement - the contact with the touch screen - is considered.

Furthermore, precision is also increased through an increased friction on the screen surface and through this generated dumping effect, physically reducing tremor deviation. The chosen touch screen may either serve as an input only device or also as output source, meaning the input application will only be displayed on the screen when interaction is required.

\section{Algorithmic Implementation}

The process of one swabbing input has been identified as a three step movement. Initiated by the first "touchdown event", every other touchdown, within certain limits, will be included in the collection of the input, due to the nature of tremor deviation. The third step is the disengagement of the user's finger from the touch screen. 
Aggregating the collected data of the whole input pattern (direction, velocity, starting point and drift) an approximation for the volitional input of the user can be accomplished. The target item will be identified regardless of whether the input finger actually directly hits the desired input location or misses it.

\subsection{Regression Analysis}

A swabbing motion is noted as a selecting action when a specific Euclidian distance is reached. We choose a value of 250 pixels $(5.25 \mathrm{~mm})$ for our user study because it is the half of the average of distance from the center of the screen (starting point) to the target (shortest distance: screen edge, longest distance: screen corner). Therefore if a touch point is more than 250 pixels away from the first touch point of the swapping motion a regression line can be calculated which runs through the desired input.

This means that all collected touch points are used to calculate a line using linear regression. To increase the numerical stability of this method we decided to use every point several times for the calculation. After that the two intersection points of the line and the area lines are identified. By transforming the line into a vector the selected symbol is found.

To prevent false touches - like other unintentional touches on the screen during a motion that are caused by jerks or extreme tremor activities - a distance per time function is used that checks if a touch point is only a specific distance away from the last coordinates regarding this time slot. During early pretests was determined that tremulous users lifted their finger for a short amount of time during their swabbing motions because of their tremor. As a consequence the user had to start the whole motion again. To ensure that short and unintentional lifts of the finger are not recognized as a touch up event, a watchdog is used. If a user lifts his/her finger from the screen the watchdog is started. If a touchdown event occurs the watchdog is stopped and reset (Fig. 2).

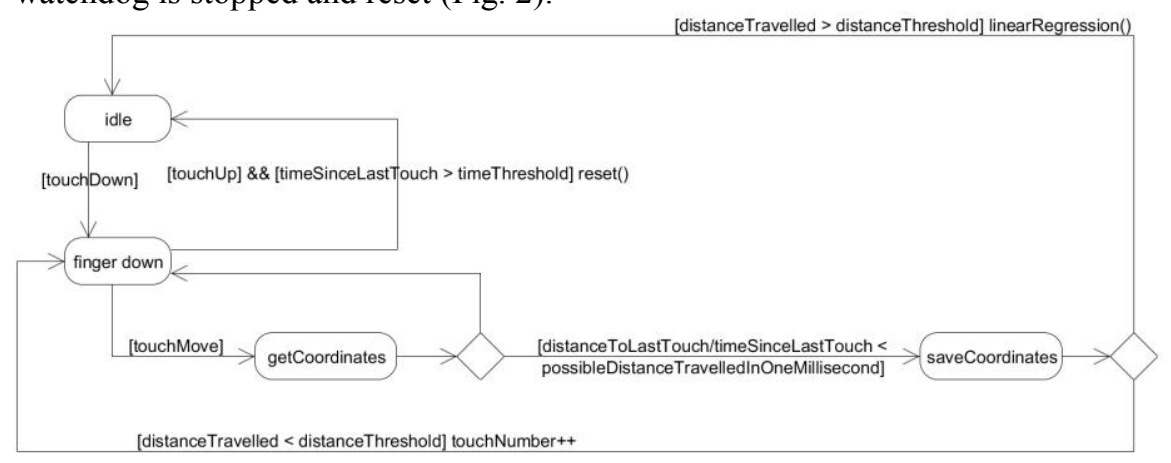

Fig. 2 Activity diagram of the algorithmic processing logic 


\subsection{Visual Representation and Feedback}

The visualization of the areas is done by arranging them circular on the screen. There are two rationales for the layout: (1) it solves the problem of overshooting a target and (2) it maximize opening angle for a given number of targets and screen space. To ensure an equal distribution this is done by rotating two points throughout the screen. By connecting this points lines are formed which are extended to the screen border. These lines are used to assist the user by giving them a visualization of an areas corresponding "corridor". To prevent users from having the feeling of crossing barriers while they make a swabbing motion, the "corridor lines" are dashed and a circle in the middle of the screen is left completely free. The points where these "corridor lines" hit the border of the task area define the boundary points of the "area line" of each area, which is needed for the calculation. To indicate which area should be activated next and whether the selection of an area was right or wrong we use polygons in the form of arrows. These arrows are filled with colors as follows:

- Blue: The area that is corresponding to the arrow should be selected

- Green Blinking: Selection of the right area

- Red Blinking: Selection of the wrong area

\section{Methods}

\subsection{Study Design}

Testing the generated swabbing input method will face the test person with the following setup: A standard multi touch notebook is placed in front of the user, using a holding frame which enables an angular positioning $\left(20^{\circ}\right.$ from desk surface $)$ to suit the test persons needs relative to the desk height [Müller-Tomfelde et al. 2008]. The probands chair will be adjusted so that the table height is approximately similar to the elbow height while the arm points towards the ground. On the screen the test person will encounter highlighted items he or she will have to select in order to perform the task (Fig. 3, left). The test person is asked to perform an "input" and will receive a visual feedback if the input was correctly performed. The interaction area is a square of $800 \times 800$ pixels (164 mm each side), and the participants have to rest the test finger on a crosshair at the same side of the hand used after each input.

The items are arranged circular (tapping \& swabbing) or in grid layout (tapping) on the screen (Fig. 4). The trials are accomplished with rising resolution starting with 9 items, 16 items and finally 25 items. Each condition is repeated for 10 trials resulting in total 90 trials. The user starts by parking the finger on the crosshair before either tapping or moving the finger to the center of the screen to swab. 


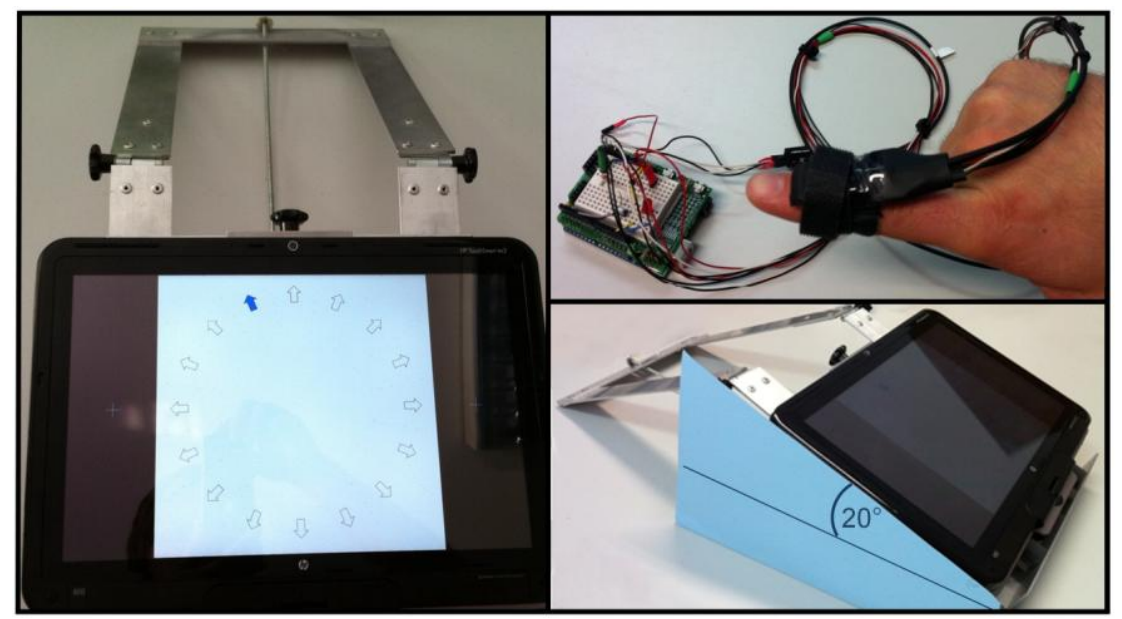

Fig. 3 Employed hardware and setup for evaluation

The screen manipulation will be done solely by the finger, no other input tool, e.g. stylus, was used. For introduction and to minimize the influence of learning effects the probands got a short hands-on demonstration for each of the three different layouts with a maximum of ten inputs per layout. Subsequently an accelerometer and gyroscope were attached to the input finger (Fig. 3, top-right) to measure oscillation for different interaction techniques as previously described by Graham [Graham 2000].

As a reference to the swabbing movements, the probands had to perform typical tapping movements (holding, resting and press-release finger moves). We decided not to provide any visual feedback to get the basic finger movement data.

Demographic factors, satisfaction about usability, cognitive and physical load as well as computer literacy were determined posterior with the help of an interviewadministered questionnaire.
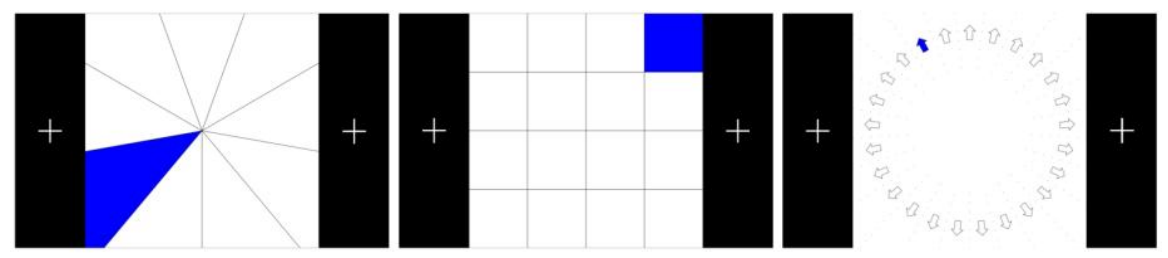

Fig. 4 Different layouts for the selection tasks with rising resolution (from left to right) radial-tapping, 9 items; grid-tapping, 16 items; swabbing, 25 items

To guarantee comparability of the results a high consistency during each individual test was conducted. A standardized test protocol helped to achieve this (Fig. 5). To prevent learning effects, we counterbalanced the order of test patterns with even-size Latin Square. 


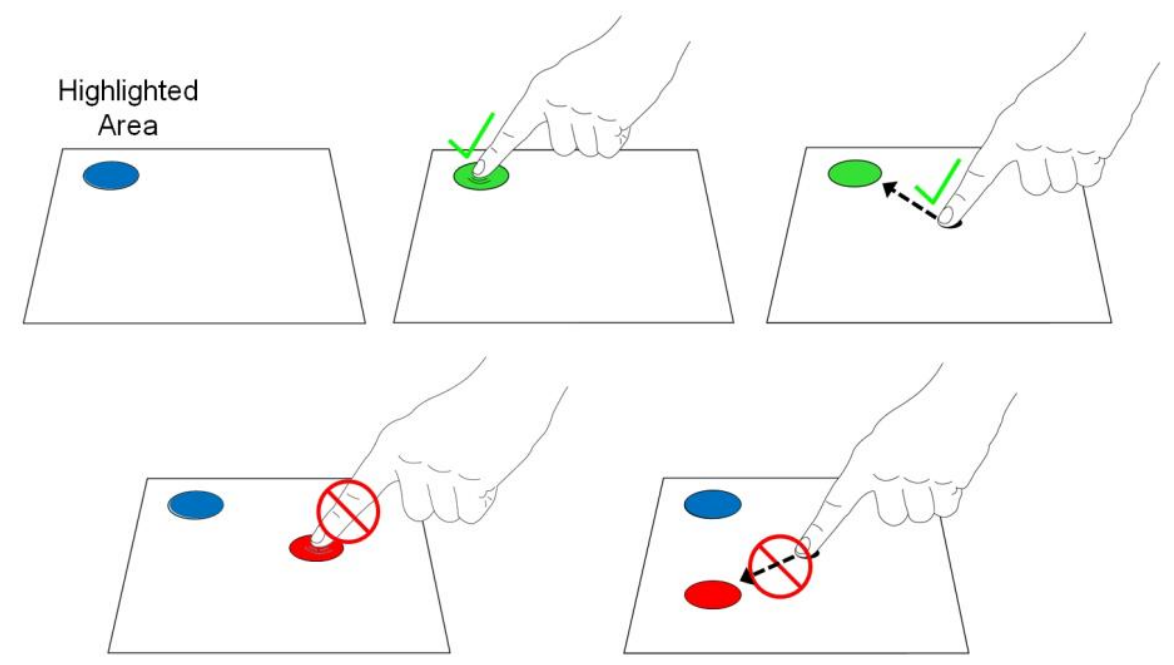

Fig. 5 Schematic illustration for introduction to the different input metaphors

\subsection{Apparatus}

The hardware platform is a HP TouchSmart tm2-1090eg with a 12.1 inch capacitive multi-touch screen $(1280 \times 800 \mathrm{px})$. The notebook was converted into tablet mode and fixed into a customized stand. No keyboard was apparent for the participant. To measure the tremor during the interaction, we attached a tri-axis accelerometer (GForce3D-3) and a gyroscope (InvenSense IGT-3200) on the backside of the extreme joint (distal phalanges) of the test finger by a Velcro ring (Fig. 1). When the finger rests on the screen, the accelerometer's $Z$ axis is orthogonal to the screen's plane, and $\mathrm{X}$ and $\mathrm{Y}$ axes are parallel to the respective screen axes. The equipment leaves the entire tip of the finger uncovered.

The sensors are connected with an Arduino Duemilanove which feeds the acceleration data directly to the Universal Serial Bus controller of the notebook. The data is associated with touch signals from the screen. We have made sure that the cable does not prevent the user from freely moving the hand, the arm, or any fingers.

\subsection{Spiralometry: Graphimetric Classification of Tremor Strength [Kraus and Hoffmann 2010]}

In order to adequately test tremor behavior of the target group, a new computerized assessment method has been chosen: spiralometry. As also recommended by the Movement Disorder Society (MDS) [Deuschl et al. 1998], drawing spirals supports the quantitative subjective evaluation of tremor amplitude. The computerized assessment of these spirals represents a blind and standardized metric mea- 
surement which is independent from subjective judgment from the investigator as well as examination's time and location. The clear guidelines of this method make it very objective and the easy setup of the experiment enables a swift process of the test person. The investigator only needs a paper and a pencil during the test, which makes the implementation of the test very feasible. The test person is asked to draw two spirals, one with each hand (Fig. 6). It is essential that the test person does not rest his writing hand and the corresponding forearm on the table. Each drawing will then be scanned by a standard scanning hardware, measuring the amplitude of each spiral in millimeters. One of important disadvantage of the paper-pencil-version is certainly the loss of time as basic information for determination of frequency. Although the pristine use of spiral drawing analysis was the control of therapy effects, it now is a useful design for capturing the current tremor amplitude of the participants when conducting the user study.
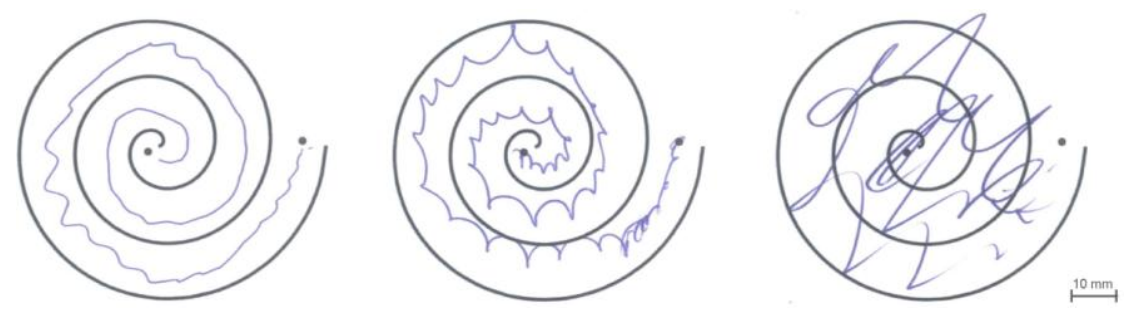

Fig. 6 Exemplarily spirals from persons with different tremor strengths

\subsection{Participants}

15 clinically diagnosed tremor patients were recruited from the Department of Neurosurgery at the University Hospital of the Aachen University (age: $\min =56$, $\mathrm{q} 1=67$, med $=75$, mean $=73,56, \mathrm{q} 3=78, \max =83$ ).

The participants had not used touch screens before this study. All participants used the index finger of their dominant hand in the experiment (Table 1).

Table 1. Exemplarily tremor profiles of some participants and interaction stages that tremor is most intense (see: section 5.3), axes of tremor, and effect of swabbing that lessens/worsens the tremor compared to press-release. (The axes are ordered by tremor strength from high to low. Missing values means insignificant effect.)

\begin{tabular}{llcccccc}
\hline \# & Tremor strength & $\begin{array}{c}\text { Dominant } \\
\text { hand }\end{array}$ & Gender & $\begin{array}{c}\text { Most intense } \\
\text { stage }\end{array}$ & Axes & Lessen & Worsen \\
\hline 1 & severe $>2 \mathrm{~cm}$ & $\mathrm{R}$ & $\mathrm{M}$ & Rest & $\mathrm{Y}, \mathrm{Z}, \mathrm{X}$ & & $\mathrm{Z}, \mathrm{Y}, \mathrm{X}$ \\
2 & slight $<0.5 \mathrm{~cm}$ & $\mathrm{R}$ & $\mathrm{M}$ & Over & $\mathrm{Y}$ & $\mathrm{X}, \mathrm{Z}$ & \\
3 & marked $1-2 \mathrm{~cm}$ & $\mathrm{R}$ & $\mathrm{M}$ & Over & $\mathrm{Y}, \mathrm{Z}$ & $\mathrm{X}, \mathrm{Z}$ & \\
4 & moderate $0.5-1 \mathrm{~cm}$ & $\mathrm{~L}$ & $\mathrm{~F}$ & Over & $\mathrm{X}, \mathrm{Z}$ & $\mathrm{X}, \mathrm{Z}$ & \\
5 & severe $>2 \mathrm{~cm}$ & $\mathrm{R}$ & $\mathrm{M}$ & Over & $\mathrm{Y}, \mathrm{Z}$ & $\mathrm{X}, \mathrm{Z}$ & $\mathrm{Y}$ \\
\hline
\end{tabular}




\section{Results}

Immediate, several observations without further scientific evaluation lead to the following findings: Persistent contact with the touch screen already significantly reduced finger oscillation relative to the reference test of tapping movements for most tremor types. As also previously found by Schneider et al [Schneider et al. 2008], a touch screen serves as a very decent tool of interaction with elderly and those who have only few experiences with computer technology. This is an indication of touch screen technology being an ergonomic tool for interaction for the target group.

\subsection{Attitudes towards Computers}

Based on the works of Gina et al. [Gina and Sherry 1992] the attitudes of elderly people suffering from tremor towards computers were evaluated a priori. The 15 strictly positive formulated statements were rated according to a 4-point Likert Scale (Fig. 7). The correlation between the user's attitude towards a system and his /her effectivity and satisfaction is unquestioned in the field of working environments. Our results with the focus on personal assistance and aids for disabled people affirm the coherence in terms of relation between acceptance of assistive technology and the specific training time and success rate.

These parameters are of special importance when applying the swabbingtechnique in (tele)medical scenarios, as first contact is usually created within the conditions of a medical necessity, rather than voluntarily.

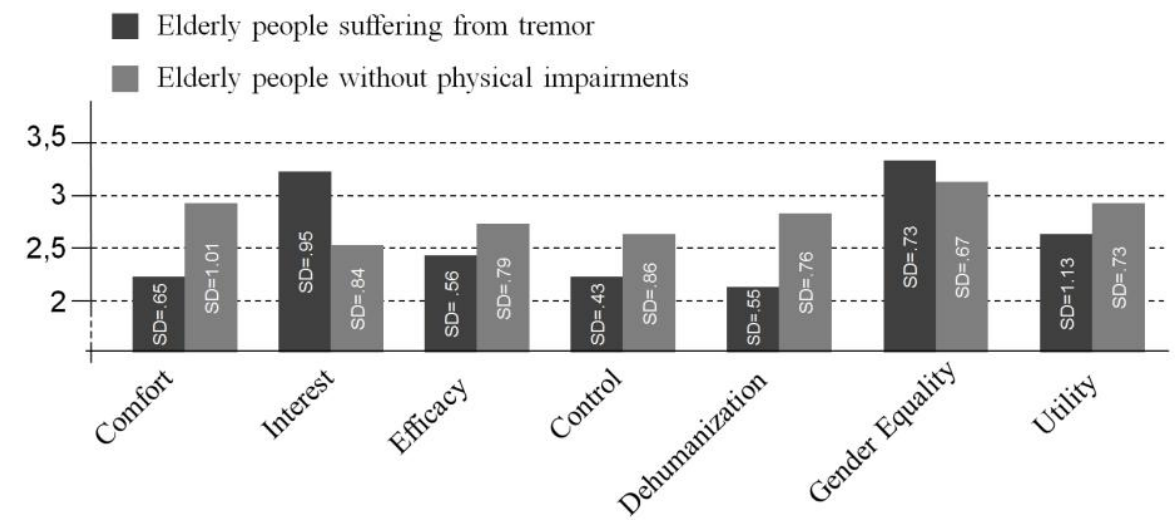

Fig. 7 Attitudes of elderly people towards computers; comparison between people with and without tremor symptoms 


\subsection{Computer Literacy}

In order to measure computer literacy and evaluate the correlation with the test results a questionnaire from Sengpiel et al. [Sengpiel et al. 2008] with nineteen items was used. The survey is based on the symbol and term knowledge gained during human computer interaction and is generally seen as an indicator towards a person's competence in handling a computer system.

The test showed that persons with an computer handling experience of more than 7 years in average completed the trials $37 \%$ faster and made $19 \%$ less input errors. Here it was insignificant, if the usage was in a private environment or job related. $71 \%$ of the interviewees said they are not using a computer more than three times a week. As main reasons were termed the deficient usability (Software \& Hardware) and unavailability.

\subsection{Learning Curve}

The results of a previous evaluation with 20 elderly first time users without tremor symptoms showed a steep learning curve for the self trained adaption of the swabbing input technique (Fig. 8). A time-stable input was reached after 20 to 22 input cycles with an orientation phase from cycles 1 to 6 . The average time for each input after cycles 20-22 lay at 1.52 seconds. This is a $100 \%$ input time reduction if compared to the initial input, supporting the steep learning curve argument and Schneider et al. The rise in productivity and quality (lower error ratio) are prime factors in the increase of success.

The input time for tapping input from the test persons showed a consistency after cycle 6 already. This proves the existence of a mature mental model on the standard approach of button use which for swabbing inputs yet has to evolve (Fig. 8).

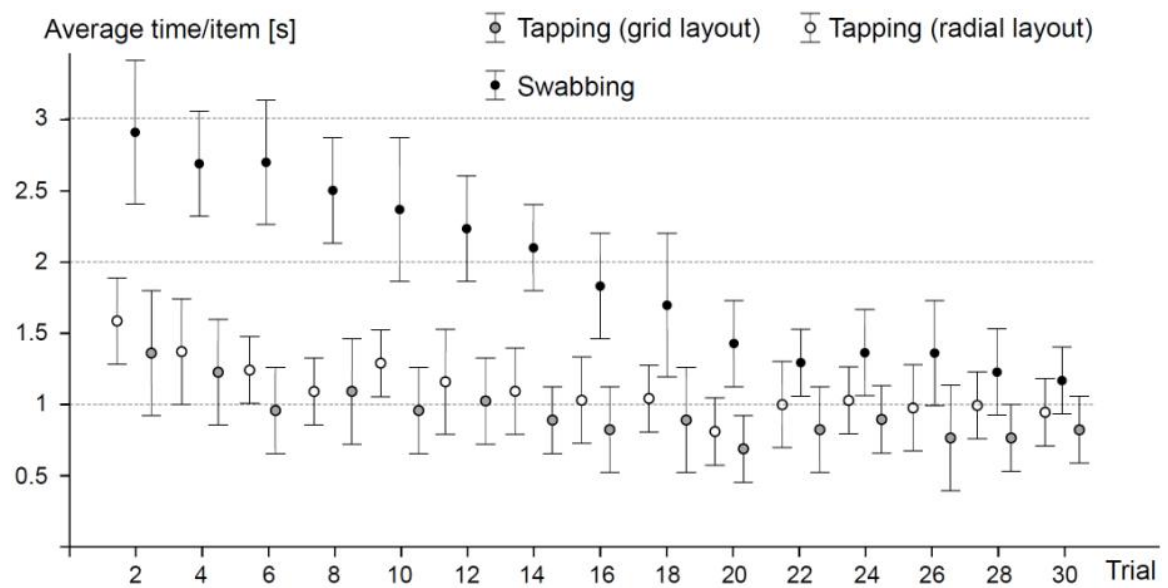

Fig. 8 Learning curve for elderly first time users for tapping/swabbing input on a touch screen 


\subsection{Parameters Influencing Error Ratio}

In the following results, we used two-way, repeated measures ANOVA models with significance level of $\alpha=0.05$; data is normally distributed. We found no interaction between layout and resolution $(F(2,35)=0.780$, n.s. $)$, and no significant difference between different layouts in tapping $(F(1,35)=3.128$, n.s. $)$. Then, comparing between methods in radial layout shows significant effect of methods $(F(1,37)$ $=5.707, p<.05)$. As seen in Figure 9, the error rates of swabbing in 16- and 25buttons resolutions are lower than tapping. Post-hoc analysis with pairwise t-test with Bonferroni correction supports the effect in both resolutions (16: $p=0.0065$, 25: $p=0.042$ ).

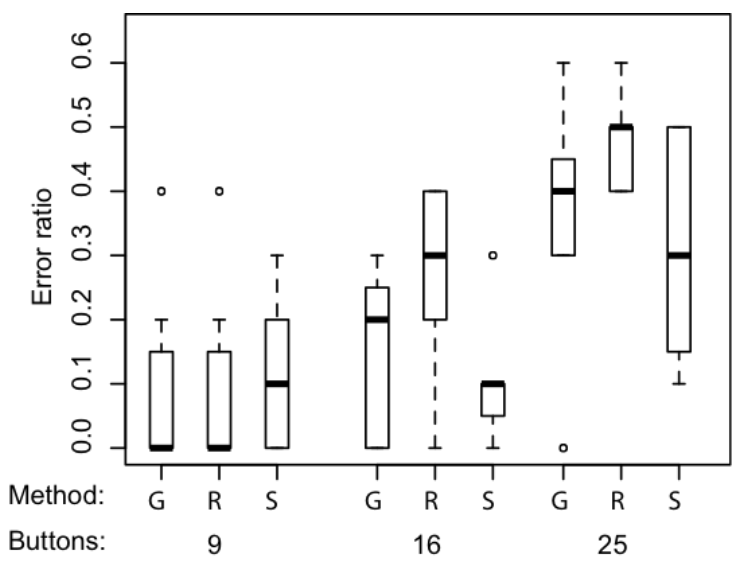

Fig. 9 Box plots of error ratio by resolutions and methods (G: tapping, grid, R: tapping, radial, S: swabbing, radial)

\subsection{Tremor Characteristics}

The acceleration data is Fast-Fourier transformed into frequency domain. This enables an analysis of tremor frequencies during rest and interaction with the touch screen. The frequency domain data can be visualized in spectrum plot. The frequency domain of data shown in Fig. 10 is presented in Fig. 11. Each plot represents frequency in an axis of a stage of interaction.

We used the highest peak that is in the tremor frequency range $(3-30 \mathrm{~Hz})$ as a tremor frequency in each axis. In an axis, if the magnitude of tremor frequency exceeds $1 \mathrm{SD}$ of the rest of frequencies in tremor range, we consider that axis a tremor axis. In Table 1, we listed the interaction stage with tremor and the respective tremor axes. Based on this data, we compared whether the tremor is worsen or lessen in swabbing compared with tapping. This result is also shown in Table 1. The investigated patterns show that measured effects are strongly dependent on the tremor type (resting, contraction, posture and intention tremor), especially re- 
garding the axes of movement on which the tremor agitation appears (see tremor profile in Table 1 for details).

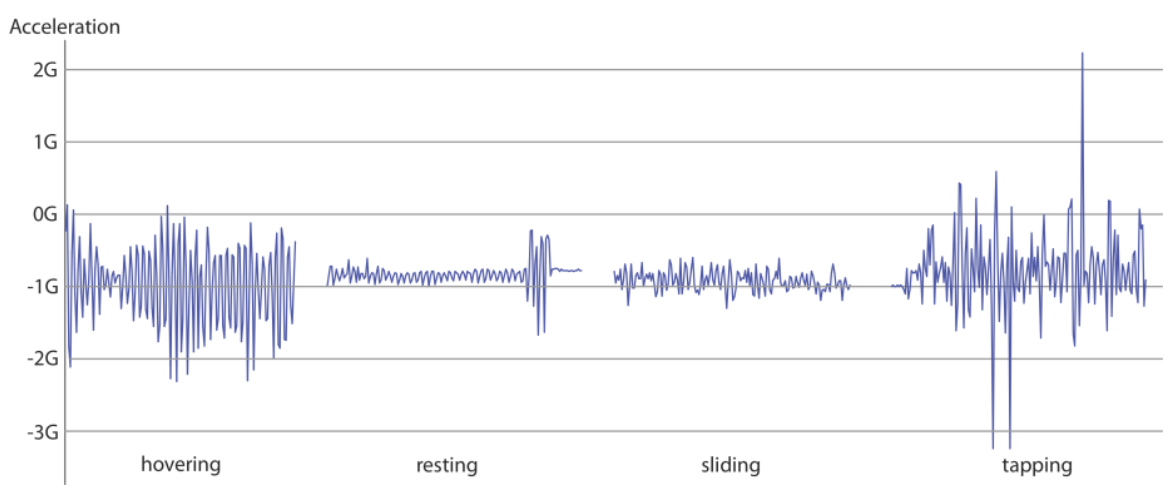

Fig. 10 An excerpt of acceleration data from each stage of interaction

The wiping movement shows the best effect for those patients suffering from an intention and resting tremor, as here deviations among the main axes of movement are reduced, while for persons with contraction tremor sliding worsens the symptoms.
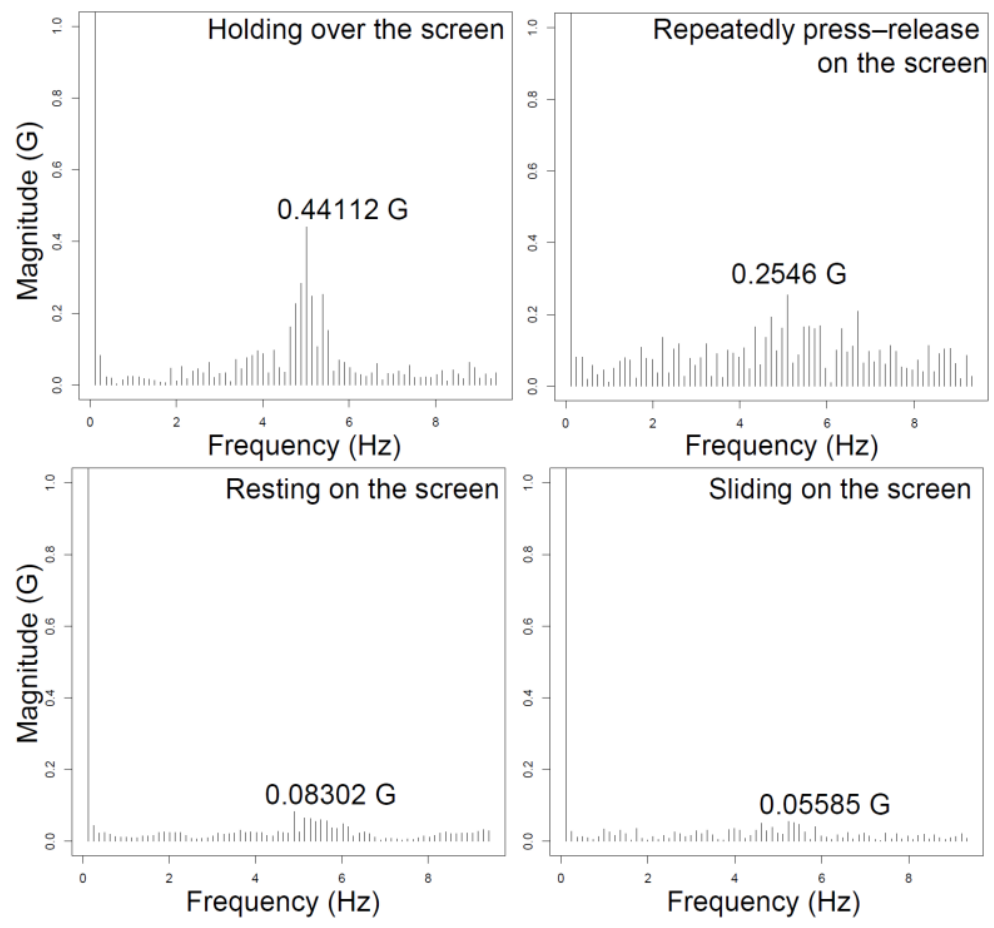

Fig. 11 Fourier plots of finger oscillation frequencies and magnitude in axis orthogonal to the ground from participant \#5. The number indicates the value of the highest peak 
The results further show, that people encumbered with medium and strong tremors, who use wiping movements as interaction, show a significantly reduced error rate of input compared to those touching standard button environments. It is also shown that patients suffering from a minor tremor agitation experience no noteworthy improvement during their interaction.

\subsection{User Satisfaction}

The satisfaction from the user while interacting with the system as well as usability was investigated by components of the "Post Study System Usability Questionnaire" (PSSUQ).

The Friedman test gave no noteworthy differences, for the given alternatives, with system satisfaction $\left(\chi^{2}=1 ;\right.$ n.s. $)$, quality $\left(\chi^{2}=2\right.$; n.s. $)$, or usability $\left(\chi^{2}=0,667\right.$; n.s.). All systems were generally evaluated positively and usage was rated as comfortable and intuitive.

\subsection{Offset Analysis for Tapping Buttons}

The evaluation of user input from participants suffering from tremor showed a significant offset for the core area of inputs depending on handedness. For analysis all button coordinates from grid layout were normalized in terms of size and positioning and the tracked contact points plotted separately for left handed and right handed touch screen users (Fig. 12).

The visualization shows that more than $90 \%$ of all fault insertions (not hitting the highlighted target area) are on the particular half side of the button of the person's dominant hand.

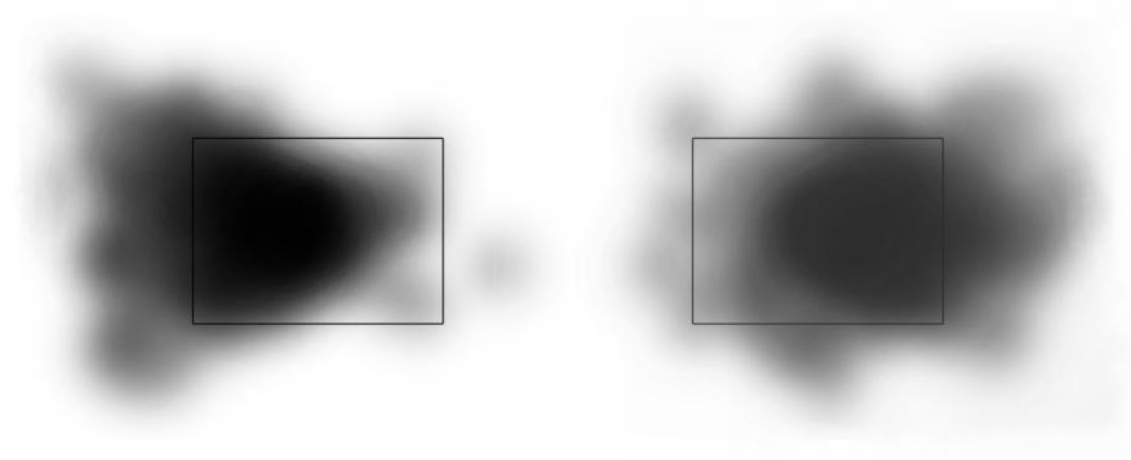

Fig. 12 Heat maps for the logged touch screen coordinates during grid-tapping, separated for left and right handed probands suffering from tremor. Higher frequency of selection is represented by darker gray tone 


\section{Discussion}

The accomplished user study suggests that swabbing input gestures reduces error ratio for touch screen selection in older tremor patients. The result shows significant advantages of swabbing for 16 targets (button width $=41 \mathrm{~mm}$ ). For 9 targets, buttons did slightly, but not significantly better. We speculated that the size of the buttons were big enough to accommodate accidental movement from tremor. For 25 targets, buttons performed consistently worse, but the high variance in the swabbing results kept this from becoming statistically significant. However, the significant result in 16 targets makes swabbing worth for further study with more participants and higher resolution. Although swabbing takes more time to input than tapping, the results do not show differences in user satisfaction. This means that the trade-off between interaction time and accuracy is acceptable for tremor patients.

We believe that swabbing will make touch screen interaction more accessible to tremor patients, especially elderly persons, in the future.

\section{Acknowledgment}

- Participants of the studies for generously helping us in our research

- $\quad$ Parkinson Patients Self-Help Group Aachen

- Federal Ministry of Education and Research and body responsible for project DLR

- German B-IT Foundation.

\section{References}

[Martínez-Martín 1998]

Martínez-Martín P (1998) An introduction to the concept of "quality of life in Parkinson's disease" In: Journal of Neurology, Springer, Berlin, pp 2-6

[Wenning et al. 2005]

Wenning GK, Kiechl S, Seppi K, Mueller J, Hogl B, Saletu M, Rungger G, Gasperi A, Willeit J, Poewe W (2005) Prevalence of movement disorders in men and women aged 50-89 years (Bruneck Study cohort): a population-based study. In: The Lancet Neurology 4 (12), pp 815-820

[Müller-Tomfelde et al. 2008]

Müller-Tomfelde C, Wessels A, Schremmer C (2008) Tilted tabletops: In between horizontal and vertical workspaces. In: Proc. TABLETOP 2008, pp 49-56

[Graham 2000]

Graham B (2000) Using an accelerometer sensor to measure human hand motion. Massachusetts Institute of Technology

[Bain and Findley 1993]

Bain PG, Findley LJ (1993) Assessing Tremor Severity: A Clinical Handbook. Smith-Gordon, London 
[Kraus and Hoffmann 2010]

Kraus PH, Hoffmann A (2010) Spiralometry: Computerized assessment of tremor amplitude on the basis of spiral drawing. In: Journal of Movement Disorders 25 (13):2164-2170

[Deuschl et al. 1998]

Deuschl G, Bain P, Brin M, Ad Hoc Scientific Committee (1998) Consensus Statement of the Movement Disorder Society on Tremor. In: Journal of Movement Disorders 13: pp 2-23

[Gina and Sherry 1992]

Gina MJ, Sherry LW (1992) Influence of Direct Computer Experience on Older Adults' Attitudes Toward Computers. In: Journal of Gerontology - Psychological Science 47 (4):250-257

[Sengpiel et al. 2008]

Sengpiel M, Struve D, Dittberner D, Wandke H (2008) Entwicklung von Trainingsprogrammen für ältere Benutzer von IT-Systemen unter Berücksichtigung des Computerwissens [Development of trainign programms for elderly users of IT-systems considering the computer literacy]. In: Wirtschaftspsychologie, Alter und Arbeit, 2008(3), pp 94-105

[Schneider et al. 2008]

Schneider N, Wilkes J, Grandt M, Schlick C (2008) Investigation of Input Devices for the Agedifferentiated Design of Human-Computer Interaction. In: Human Factors and Ergonomics Society (ed) Proceedings of the Human Factors and Ergonomics Society, Mira Digital Publishing, New York, pp 144-148 